Original research article

\title{
A biochemical approach to the anti-inflammatory, antioxidant and antiapoptotic potential of beta-carotene as a protective agent against bromobenzene-induced hepatotoxicity in female Wistar albino rats
}

\author{
Priya Josson Akkara ${ }^{1,2}$, Evan Prince Sabina ${ }^{1 *}$ \\ ${ }^{1}$ Vellore Institute of Technology, School of Bio Sciences and Technology, Vellore, India \\ ${ }^{2}$ Kristu Jayanti College (Autonomous), Bengaluru, India
}

\begin{abstract}
Bromobenzene is a compound which has contributed much in understanding the mechanisms involved in xenobiotic hepatotoxicity induced by drugs and environment pollutants. In the present study, the protective and ameliorative effect of beta-carotene was investigated against bromobenzene-induced hepatotoxicity and compared with silymarin, a standard hepatoprotective reference drug. Beta-carotene ( $10 \mathrm{mg} / \mathrm{kg}$ b.w. p.o.) was administered to the rats for 9 days before intragastric intubation of bromobenzene ( $10 \mathrm{mmol} / \mathrm{kg}$ b.w.). Liver marker enzymes (aspartate transaminase, alanine transaminase and alkaline phosphatase), total protein content, bilirubin, total cholesterol, high-density lipoproteins, triglycerides, antioxidant status (reduced glutathione, superoxide dismutase, catalase, glutathione-S-transferase and glutathione peroxidase) were assessed along with histopathological analysis. ELISA was performed for analysing the levels of cytokines such as TNF- $\alpha$, IL-1 $\beta$ and IL- 6 in serum and in the liver. Caspase-3, COX-2 and NF- $\mathrm{kB}$ were evaluated by Western blotting. Administration of bromobenzene resulted in elevated levels of liver marker enzymes, bilirubin, lipid peroxidation and cytokines but deterioration in total protein content, antioxidant levels and histopathological conditions. Pre-treatment with beta-carotene not only significantly decreased the levels of liver markers, lipid peroxidation and cytokines but also improved histoarchitecture and increased antioxidant levels minimising oxidative stress, and reduced factors contributing to apoptosis. This significant reversal of the biochemical changes on pre-treatment with beta-carotene in comparison with rats administered with bromobenzene clearly demonstrates that beta-carotene possesses promising hepatoprotective effect through its antioxidant, anti-inflammatory and antiapoptotic activity and hence is suggested as a potential therapeutic agent for protection from bromobenzene.
\end{abstract}

Keywords: Antioxidant; Beta-carotene; Bromobenzene; Cytokines; Hepatotoxicity; Oxidative stress

\section{Highlights:}

- Pre-treatment with beta-carotene increases antioxidant levels and help in maintaining normal liver functions thus combating oxidative stress promoted by bromobenzene.

- Beta-carotene safeguards liver from toxicity induced by bromobenzene due to its anti-inflammatory and antiapoptotic activity thereby sustaining the histo-architecture of liver.

- Protective effect of beta-carotene against bromobenzene induced hepatotoxicity was found to be at comparable levels to that of standard hepatoprotective drug, silymarin.

- The hepatoprotective effect of beta-carotene makes it a potential therapeutic candidate for shielding against bromobenzene induced hepatotoxicity.

- Beta-carotene could be used as a protective agent for prevention of liver injury caused by environment pollutants.

\section{Introduction}

One of the primary health concern in today's world is toxicity caused by exposure to a variety of pollutants in the environment that induce oxidative stress. Oxidative stress plays a vital role in the pathogenesis of chemical and drug-induced chronic liver diseases (Ezhilarasan, 2018). Although humans have developed a network of antioxidant systems that guard cells from pro-oxidant conditions, excessive reactive species derived from oxygen and nitrogen might cause oxidative injury to tissues and organs. Administration of antioxidants indicates to be a logical preventive and curative strategy for tackling oxidative stress related liver diseases. The toxic effects of drug-induced hepatotoxicity was found to be decreased by agents with antioxidant activity in experimental studies on

\footnotetext{
* Author for correspondence: Evan Prince Sabina, Vellore Institute of Technology, School of Bio Sciences and Technology, Vellore 632014, Tamil Nadu, India; e-mail: eps674@gmail.com http://doi.org/10.32725/jab.2020.011

Submitted: 2019-02-21 • Accepted: 2020-07-28 • Prepublished online: 2020-08-10

J Appl Biomed 18/2-3: 87-95 • EISSN 1214-0287 • ISSN 1214-021X

(C) 2020 The Authors. Published by University of South Bohemia in České Budějovice, Faculty of Health and Social Sciences.

This is an open access article under the CC BY-NC-ND license.
} 
rats (Feyissa et al., 2013). The health benefits of innumerable medicinal and edible plants is due to the anti-inflammatory, antioxidant and free radical scavenging activities of the natural antioxidants they possess (Li et al., 2015). Studies show that higher dietary intake of antioxidant vitamins may reduce the risk of toxicity (Ma et al., 2018).

Bromobenzene (BB) is a xenobiotic that is released into the environment during its production in industries and is known to induce hepatotoxicity. It is generally used as an additive in motor oil and as a solvent for large scale crystallizations (Hamed et al., 2013; Heijne et al., 2004). Exposure to BB leads to the accumulation of many reactive secondary metabolites of BB in the cells, leading to elevated levels of free radicals and reduction in the antioxidants. Thus, the obstruction to the generation of free radicals via antioxidant activity plays an important role in reducing toxicity (Vedi et al., 2014a).

Beta-carotene $(b-c)$ is a prospective antioxidant and belongs to the class of carotenoids (Wang and Russell, 1999). Their absorption may be associated with interception of cancer, heart diseases, and age linked macular degeneration (Nita and Grzybowski, 2016). Beta-carotene has shown promising anti-inflammatory activity in RAW264.7 cells, perhaps as a result of their ROS-scavenging activity (Fujisawa and Murakami, 2016). Beta-carotene was found to decrease stereotyped behaviours and to enhance social interactions in autistic-like behaviour in mice (Avraham et al., 2017). Increased intake of dietary beta-carotene, selenium, vitamin $C$, vitamin $\mathrm{E}$ and $\beta$-cryptoxanthin decreased the risk of pancreatic cancer (Chen et al., 2016; Koriem and Arbid, 2018). Amelioration of cardiotoxicity was observed in rats on treatment with beta-carotene (Bahadir et al., 2018). It was recommended to consume food rich in beta-carotene and retinol in areas highly polluted with polycyclic aromatic hydrocarbons (Darwish et al., 2018). However, avoiding oxidant sources such as cigarette smoke and alcohol must be considered when taking dietary antioxidants (Liu et al., 2018).

The existing options for treating hepatic pathologies have limited advantages along with complications. Therefore, there is an urgent need for better agents and different approaches for the prevention and treatment of xenobiotics-induced hepatotoxicity. Although silymarin (SLY) and N-acetyl-L-cysteine which possess strong hepatoprotective activity are being used in the clinical therapy for liver injury, some of the complications due to their administration are restricted efficacy, gastric irritation and allergies (Fried et al., 2012). This indicates that there is still a need for finding highly effective and reliable drugs with minimal side effects for the prevention of acute liver failure caused by xenobiotics. The present study aimed to assess the prospective protective role of beta-carotene against BB-induced hepatotoxicity in female Wistar albino rats. The standard drug SLY was used as a reference drug for the purpose of comparison.

\section{Materials and methods}

\section{Chemicals and reagents}

Bromobenzene was purchased from Sigma Chemical Company. Commercially available beta-carotene was purchased from Natural Remedies, Bangalore, India. Silymarin, a standard hepatoprotective drug was purchased from Micro Labs Ltd., Goa, India. Tumour necrosis factor-alpha (TNF- $\alpha$ ), Interleukin-1beta (IL-1 $\beta$ ) and Interleukin-6 (IL-6) were measured using commercial ELISA kits from Sigma Aldrich, Bangalore, India. The antibodies against Caspase-3, Cyclooxygenase-2
(COX-2) and Necrosis factor kappa B (NF- $\mathrm{B}$ ) for Western blotting were purchased from Taurus Inc., India. Commercial diagnostic kits for aspartate amino transferase (AST), alanine amino transferase (ALT), alkaline phosphatase (ALP), total and direct bilirubin, total cholesterol, high density lipoprotein (HDL), total protein and albumin were procured from AutoSpan Diagnostics Ltd., Surat, Gujarat, India. All the other chemicals and reagents used were acquired locally and of analytical grade.

\section{Animals}

Thirty female Wistar albino rats weighing about 120-150 g and 6-8 weeks old were obtained from the animal house of VIT University, Vellore, Tamil Nadu, India. They were accustomed for a week before the onset of the experiment. The rats were housed six in each cage and maintained in a temperature and light controlled room. The animals were allowed to freely acquire water and commercial pelleted rat feed procured from Hindustan Lever Ltd., Mumbai, India. The experimental procedure was approved by the institutional ethical committee, VIT University, Vellore, India (VIT/IAEC/13/Feb13/20). Rats were allocated randomly into five groups of six each and were treated as follows (Gopi and Setty, 2010). The effective dosage of BB and beta-carotene (Vedi et al., 2014a, b) was decided on the basis of previous studies and $10 \mathrm{mmol} / \mathrm{kg} \mathrm{b.w.} \mathrm{of} \mathrm{BB} \mathrm{was}$ dissolved in coconut oil before administration. $25 \mathrm{mg} / \mathrm{kg}$ b.w. of silymarin and $10 \mathrm{mg} / \mathrm{kg}$ b.w. of beta-carotene were administered to rats by preparing an aqueous suspension in double distilled water.

Group 1: Normal control was administered with $0.1 \mathrm{ml}$ coconut oil/day, by intragastric intubation only once on the ninth day.

Group 2: Administered with BB, $10 \mathrm{mmol} / \mathrm{kg}$ b.w. in $0.1 \mathrm{ml}$ coconut oil by intragastric intubation once on the ninth day.

Group 3: Administered with beta-carotene, $10 \mathrm{mg} / \mathrm{kg}$ b.w./ day, p.o. for 9 days and a single dose of BB, $10 \mathrm{mmol} / \mathrm{kg} \mathrm{b.w.} \mathrm{in}$ $0.1 \mathrm{ml}$ coconut oil by intragastric intubation on the ninth day. Group 4: Administered with silymarin, $25 \mathrm{mg} / \mathrm{kg}$ b.w./day, p.o. for 9 days and a single dose of BB, $10 \mathrm{mmol} / \mathrm{kg}$ b.w. in $0.1 \mathrm{ml}$ coconut oil by intragastric intubation on the ninth day. Group 5: Administered with beta-carotene, $10 \mathrm{mg} / \mathrm{kg}$ b.w./ day, p.o. for 9 days.

The animals were sacrificed after the last dosage using ether anesthesia. Liver samples were taken for histopathological examination. Blood samples were collected and centrifuged at $2000 \mathrm{rpm}$ for $10 \mathrm{~min}$ and the serum thus separated was used for further analysis. Approximately, 0.05-0.1 g of liver tissue from samples were homogenized in phosphate buffer of $\mathrm{pH} 7.4$ to give $20 \% \mathrm{w} / \mathrm{v}$ homogenate. This homogenate was centrifuged at $3000 \mathrm{rpm}$ at $4{ }^{\circ} \mathrm{C}$ for $10 \mathrm{~min}$ and the supernatant was stored at $-20^{\circ} \mathrm{C}$ until analysis.

\section{Evaluation of hepatoprotective activity}

Serum of the experimental rats were used to determine the levels of ALP, AST, ALT, total bilirubin, direct bilirubin, total protein, albumin, total cholesterol, triglycerides and HDL using commercial diagnostic kits and assays were carried out according to the manufacturer's protocol.

\section{Analysis of antioxidant status}

Liver homogenate in $0.1 \mathrm{M}$ phosphate buffered saline (PBS) was used for the analysis of antioxidant activity like superoxide dismutase (SOD) (Marklund and Marklund, 1974), catalase (Sinha, 1972), glutathione-S-transferase (GST) (Habig et 
al., 1974), glutathione peroxidase (GPx) (Rotruck et al., 1973), reduced glutathione (GSH) (Moron et al., 1979) and thiobarbituric acid reactive substances (TBARS) (Ohkawa et al., 1979).

\section{Assessment of histopathological changes}

Liver procured from the experimental animals was washed with $0.1 \mathrm{M}$ ice cold PBS. A portion of the liver was fixed in $10 \%$ formalin and analysis of histopathological changes were carried out after staining the tissue sections thus processed with haematoxylin and eosin.

\section{Analysis of cytokines in liver and serum}

The supernatant obtained after centrifugation of liver homogenate was used for analysis of cytokines in liver. The concentrations of TNF- $\alpha$, IL-1 $\beta$ and IL- 6 in liver and serum were measured by ELISA according to the manufacturer's protocol and were expressed in picograms per milliliter.

\section{Evaluation of protein expression}

The levels of Caspase-3, COX-2 and NF- $\mathrm{BB}$ in liver tissue homogenate and serum were evaluated using Western blotting. The total protein content was extracted from liver homogenate and serum and characterized using $8 \%$ SDS-PAGE. The proteins were transferred to polyvinylidene difluoride membranes and kept at $4{ }^{\circ} \mathrm{C}$ overnight. The samples were blocked with PBS and were incubated with primary antibodies overnight. Quantitative analysis of thus detected immune reactive proteins was performed by immunoblotting with the respective antibodies and the density of the bands was measured us- ing densitometric analysis. The results of the Western blotting analysis were expressed as the ratio of target gene with the house keeping gene.

\section{Statistical analysis}

Values were presented as mean \pm standard error (SE). Oneway analysis of variance (ANOVA) was performed using Graph Pad InStat3 software for further statistical analysis. StudentNewman-Keuls test was followed to determine the significant $\left({ }^{*} P<0.05\right)$ differences between groups.

\section{Results}

\section{Hepatoprotective effect of beta-carotene on serum liver function markers and bilirubin in BB-induced rats} Rats induced with $\mathrm{BB}$ showed significant elevation in the $\left({ }^{*} P<0.05\right)$ levels of serum liver function enzymes such as ALT, AST, ALP (Fig. 1), total bilirubin and direct bilirubin (Fig. 2). Pre-treatment with beta-carotene decreased and almost normalized the aforesaid parameters in BB treated rats when compared with that of the control. Silymarin pre-treatment also brought about a decrease in the liver function markers and bilirubin in BB-induced rats. The AST and total bilirubin levels were found to be reversed more efficiently in the beta-carotene pre-treated rats as compared to the silymarin pre-treated group. The difference in the levels of ALT, ALP and total bilirubin of beta-carotene alone treated rats compared with that of the control was not physiologically relevant.

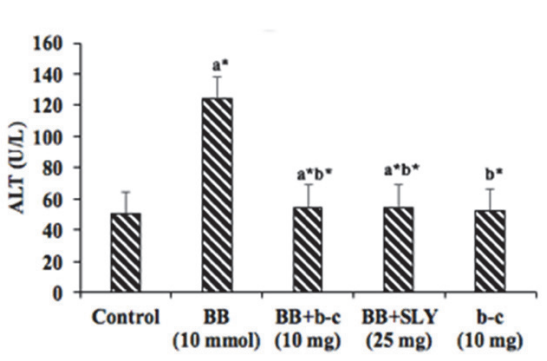

(A)

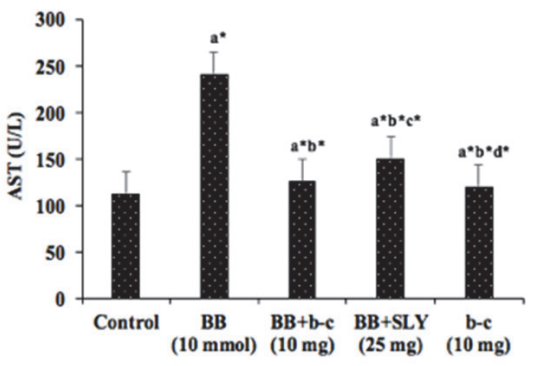

(B)

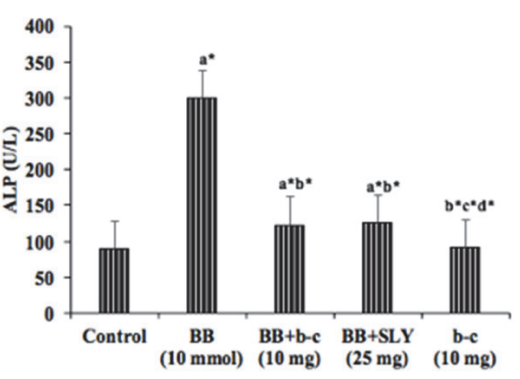

(C)

Fig. 1. Hepatoprotective effect of beta-carotene on serum liver function markers in BB-induced rats. (A) ALT, (B) AST, (C) ALP. Each value represents the mean \pm SE of six rats. Comparisons were made as follows: a - Group 1 vs Group 2, 3, 4, 5; b - Group 2 vs Group 3, 4, 5; c- Group 3 vs Group 4, 5; d - Group 4 vs Group 5. The symbols represent statistical significance at $\left({ }^{*} P<0.05\right)$. Statistical analysis was calculated by one-way ANOVA followed by the Student-Newman-Keuls test.

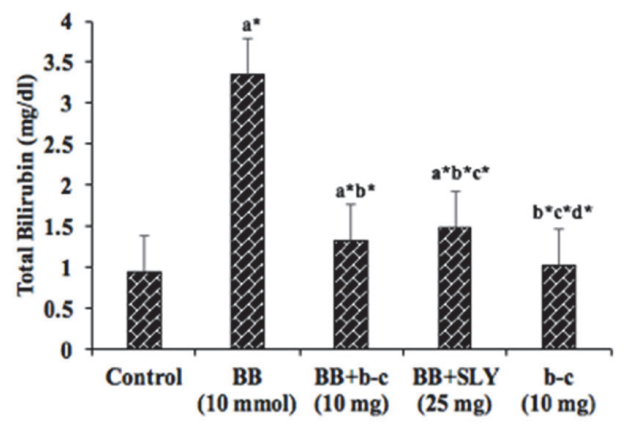

(A)

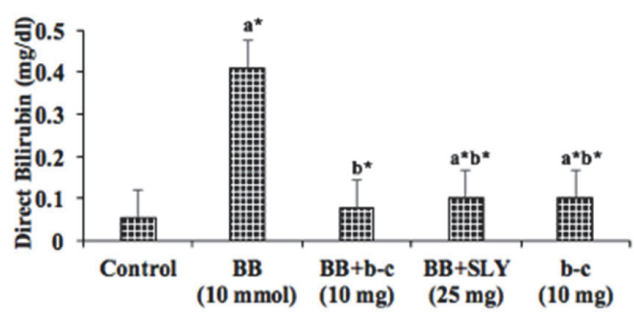

(B)

Fig. 2. Protective effect of beta-carotene on total bilirubin and direct bilirubin in BB-induced rats. (A) Total bilirubin, (B) Direct bilirubin. Each value represents the mean \pm SE of six rats. Comparisons were made as follows: a - Group 1 vs Group 2, 3, 4, 5; b - Group 2 vs Group 3, 4, 5; c- Group 3 vs Group 4, 5; d - Group 4 vs Group 5. The symbols represent statistical significance at $\left({ }^{*} P<0.05\right)$. Statistical analysis was calculated by one-way ANOVA followed by the Student-Newman-Keuls test. 


\section{Protective effect of beta-carotene on serum lipid and total protein levels in BB-induced rats}

Administration of BB produced significant $\left({ }^{*} P<0.05\right)$ hypertriglyceridemia in rats (Fig. 3 ). Pre-treatment with beta-carotene significantly decreased the elevated levels of triglycerides. (Fig. 3). The efficacy of beta-carotene in normalizing the lipid profile of $\mathrm{BB}$ treated rats was comparable to that of the sily- marin pre-treated rats. There was a significant $\left({ }^{*} P<0.05\right)$ decrease in the levels of total protein and albumin in BB-induced rats which was reversed in rats pre-treated with beta-carotene as well as for those pre-treated with silymarin (Fig. 4). The total cholesterol and total protein levels were found to be almost similar for rats treated only with beta-carotene and control rats.

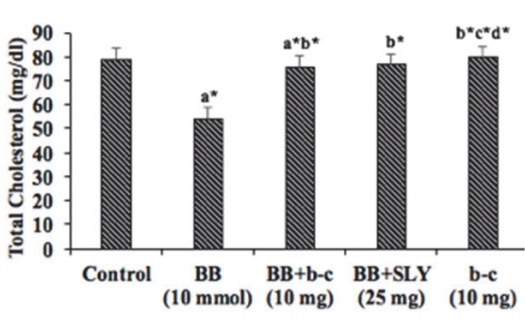

(A)

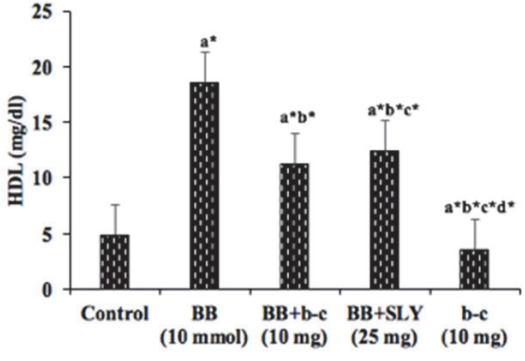

(B)

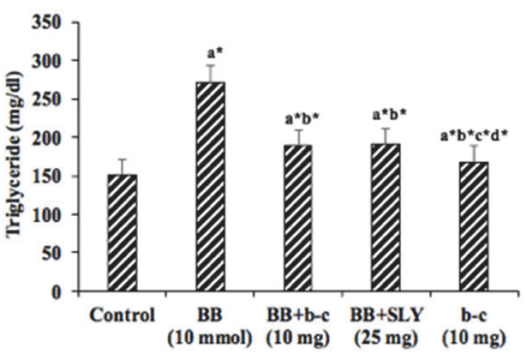

(C)

Fig. 3. Protective effect of beta-carotene on serum lipids in BB-induced rats. (A) Total cholesterol, (B) HDL, (C) Triglyceride. Each value represents the mean \pm SE of six rats. Comparisons were made as follows: a - Group 1 vs Group 2, 3, 4, 5; b - Group 2 vs Group 3, 4, 5; c- Group 3 vs Group 4, 5; d - Group 4 vs Group 5. The symbols represent statistical significance at $\left({ }^{*} P<0.05\right)$. Statistical analysis was calculated by one-way ANOVA followed by the Student-Newman-Keuls test.

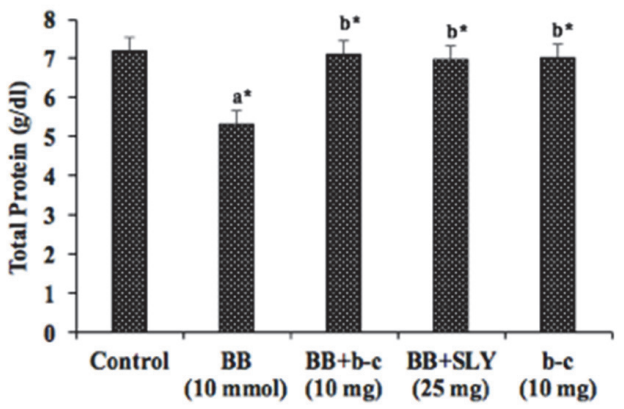

(A)

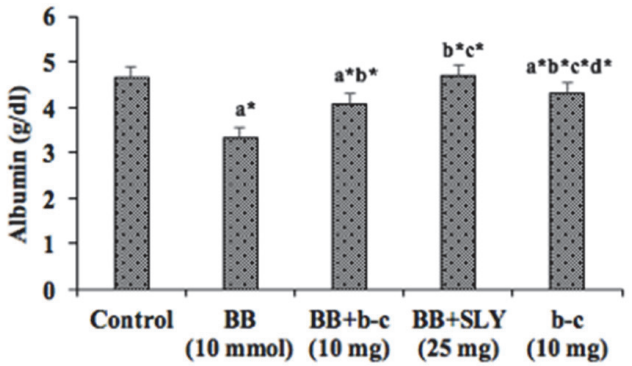

(B)

Fig. 4. Protective effect of beta-carotene on total protein and albumin in BB-induced rats. (A) Total protein, (B) Albumin. Each value represents the mean \pm SE of six rats. Comparisons were made as follows: a - Group 1 vs Group 2, 3, 4, 5; b - Group 2 vs Group 3, 4, 5; c - Group 3 vs Group 4,$5 ; \mathrm{d}$ - Group 4 vs Group 5. The symbols represent statistical significance at $\left({ }^{*} P<0.05\right)$. Statistical analysis was calculated by one-way ANOVA followed by the Student-Newman-Keuls test.

\section{Effect of beta-carotene on hepatic antioxidant status and lipid peroxidation of $B B$-induced rats}

$\mathrm{BB}$ treated rats showed significant reduction in the activities of the antioxidant enzymes, SOD, catalase, GST, GPx and hepatic glutathione content (Fig. 5). Beta-carotene pre-treatment was able to restore near normal antioxidant levels by significantly increasing $\left({ }^{*} P<0.05\right)$ the antioxidant enzymes in the liver of $\mathrm{BB}$ treated rats. The silymarin pre-treated group exhibited a similar effect in normalizing the antioxidant levels. The catalase activity in liver was effectively increased by beta-carotene pre-treatment and was found to be comparatively more than the silymarin treated BB-induced rats (Fig. 5). There was a significant increase $\left({ }^{*} P<0.05\right)$ in TBARS in the liver tissue homogenates of $\mathrm{BB}$-induced rats which was brought down effectively to normal values by beta-carotene as well as silymarin pre-treatment. The group which was treated only with beta-carotene showed normal antioxidant levels except GST similar to the control group.

\section{Hepatoprotective effect of beta-carotene on liver tissue morphology of BB-induced rats}

The sections of liver from control group showed normal histo-architecture. Hexagonal hepatocytes were arranged in a typical radiating manner with no inflammatory cells with moderate congestion in sinusoids (Fig. 6A). In the BB-induced rats of group 2, the architecture of individual hepatocytes was lost and many cells showed fragmentation of nucleus. Prominent reactive nuclei were observed along with increased sinusoidal space. Portal triad revealed mild to moderate biliary hyperplasia with cellular necrosis clearly demonstrating hepatotoxic effect of BB (Fig. 6B). In rats pre-treated with beta-carotene, hepatocytes appeared more eosinophilic but lost the pattern of cellular arrangement, with no inflammatory cells and indication of regeneration (Fig. 6C). In silymarin pre-treated rats, hepatocytes appeared more eosinophilic but lost the pattern of cellular arrangement with moderate degeneration of hepatocytes (Fig. 6D). Moderate congestion was also observed in 
SLY treated group which was not visible in Group 3, pointing out that beta-carotene might be a more efficient pre-treatment strategy for recovering from toxic effect of BB. The safety in using beta-carotene as a protective agent is shown by the normal hepatocyte arrangement with proper sinusoidal space in Group 5, which was treated only with beta-carotene (Fig. 6E).

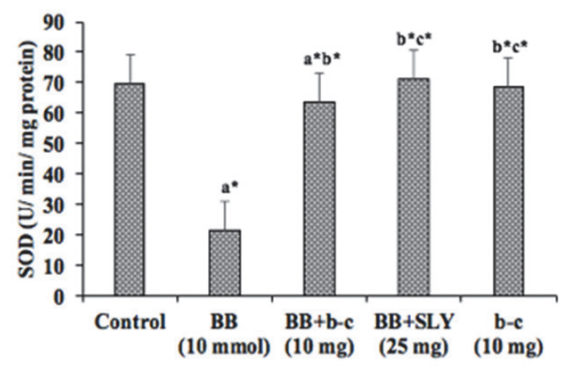

(A)

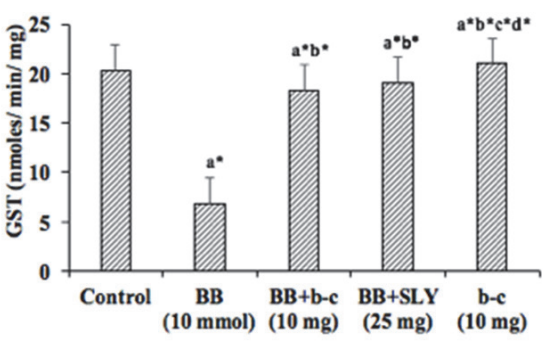

(D)

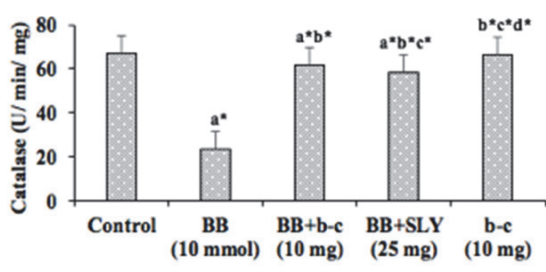

(B)

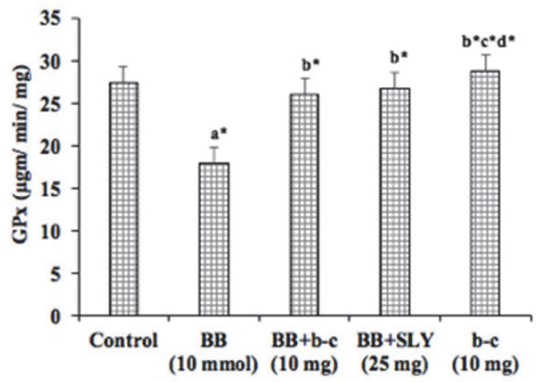

(E)

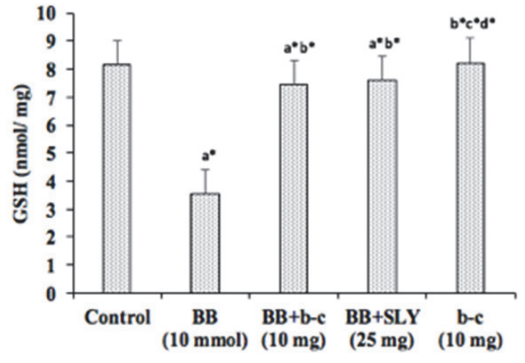

(C)

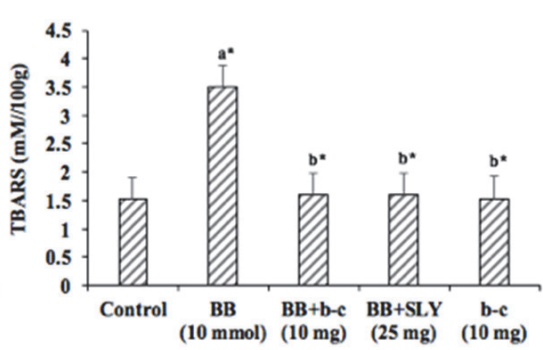

(F)

Fig. 5. Effect of beta-carotene on hepatic antioxidant status of BB-induced rats. (A) SOD, (B) Catalase, (C) GSH, (D) GST, (E) GPx and (F) TBARS. Each value represents the mean \pm SE of six rats. Comparisons were made as follows: a - Group 1 vs Group 2, 3, 4, 5; b - Group 2 vs Group 3, 4, 5; c - Group 3 vs Group 4, 5; d - Group 4 vs Group 5. The symbols represent statistical significance at $\left({ }^{*} P<0.05\right)$. Statistical analysis was calculated by one-way ANOVA followed by the Student-Newman-Keuls test.
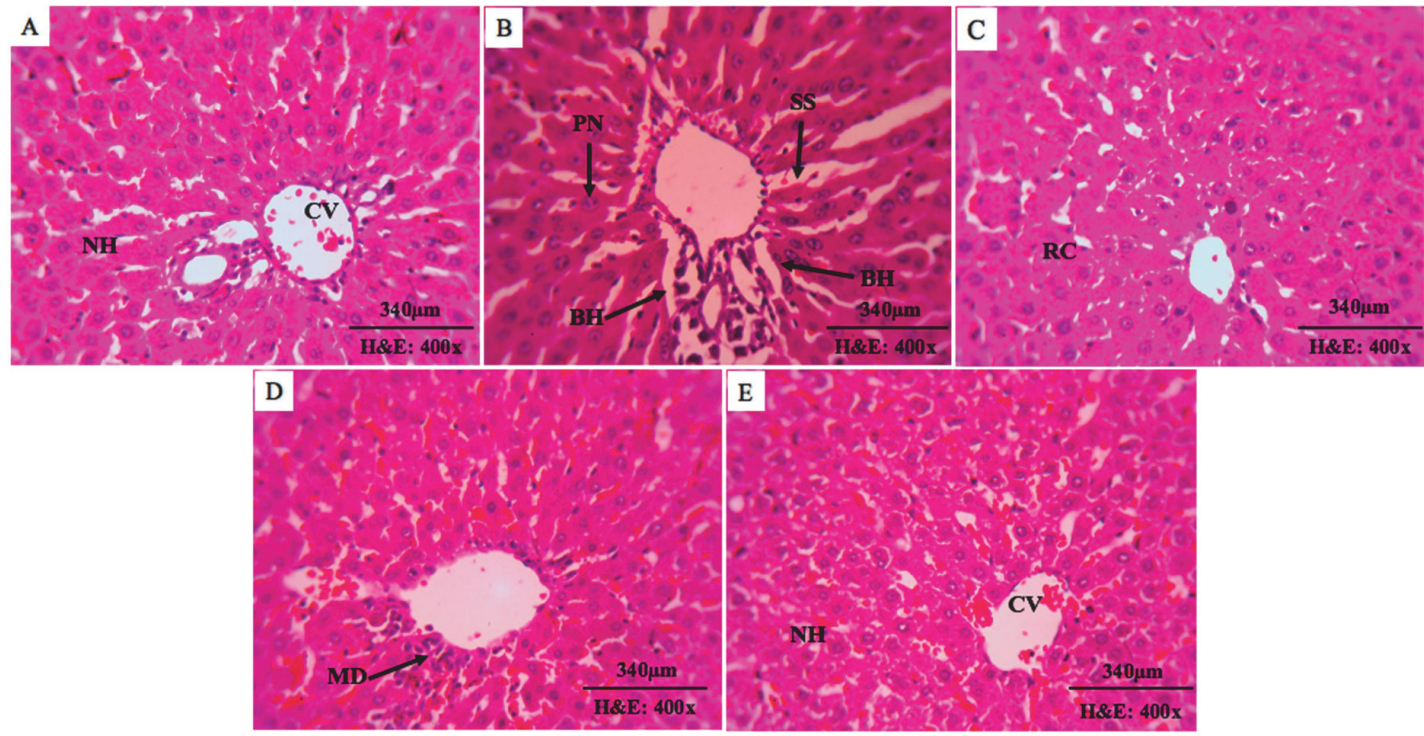

Fig. 6. Hepatoprotective effect of beta-carotene on liver tissue morphology of BB-induced rats. Photomicrography of the liver (H\&E staining): (A) Group 1 (control) showed normal histo-architecture of liver, (B) Group 2 (bromobenzene) showed prominent reactive nuclei, increased sinusoidal space, biliary hyperplaia, (C) Group 3 (beta-carotene + bromobenzene) showed no inflammatory cells, (D) Group 4 (silymarin + bromobenzene) showed moderate degeneration and (E) Group 5 (beta-carotene) showed normal hepatocytes. Normal hepatocytes (NH), central vein (CV), prominent nuclei (PN), sinusoidal space (SS), biliary hyperplasia (BH), regenerating cells (RC), mild degeneration (MD). 


\section{Effect of beta-carotene on the pro-inflammatory cytokines TNF- $\alpha, I L-1 \beta$ and IL- 6 in liver and serum of BB-induced rats}

The significant $\left({ }^{*} P<0.05\right)$ elevation in the pro-inflammatory cytokine levels TNF- $\alpha$, IL- 6 and IL- $1 \beta$ in the liver and serum of $\mathrm{BB}$-induced rats (Figures 7,8 ) indicates the association of infiltration of inflammatory cells on exposure to $\mathrm{BB}$ as with other xenobiotics. On beta-carotene pre-treatment, the TNF- $\alpha$, IL-1 $\beta$ and IL- 6 levels in liver and serum were found to be significantly $\left({ }^{*} P<0.05\right)$ reduced and the levels of liver IL-1 $\beta$, liver IL- 6 and serum IL- $1 \beta$ showed tendency to normalize to control levels due to the anti-inflammatory effect of beta-carotene. Silymarin pre-treatment elicited a similar response in reducing the pro-inflammatory cytokines as with beta-carotene treated group. The fact that beta-carotene alone does not evoke elevation in cytokines as compared to the control group confirms that beta-carotene does not cause adverse effects as a protective agent.

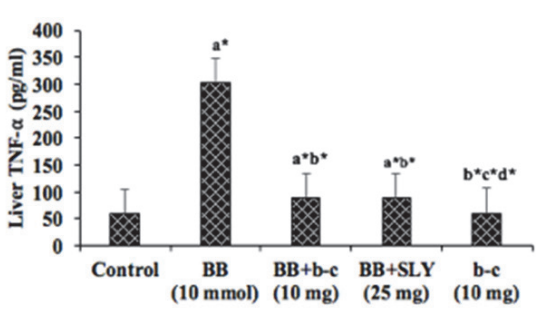

(A)

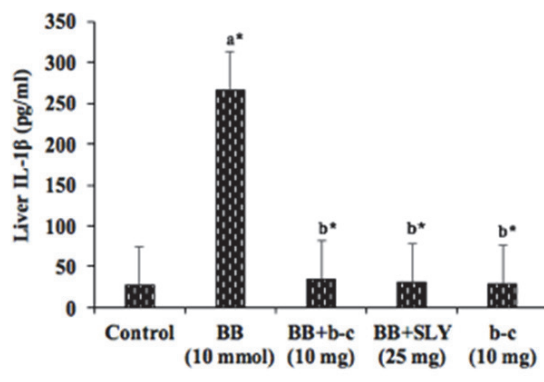

(B)

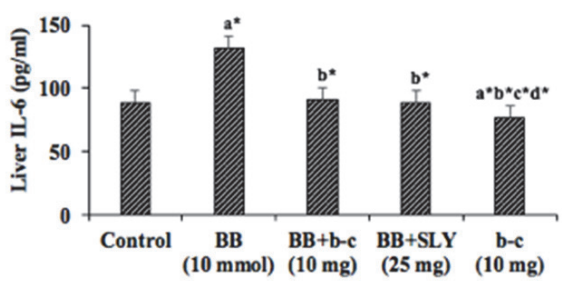

(C)

Fig. 7. Effect of beta-carotene on TNF- $\alpha$, IL-1 $\beta$ and IL-6 in liver of BB-induced rats. (A) Liver TNF- $\alpha$, (B) Liver IL-1 $\beta$, (C) Liver IL-6. Each value represents the mean \pm SE of six rats. Comparisons were made as follows: a - Group 1 vs Group 2, 3, 4, 5; b - Group 2 vs Group 3, 4, 5; c- Group 3 vs Group 4, 5; d - Group 4 vs Group 5. The symbols represent statistical significance at $\left({ }^{*} P<0.05\right)$. Statistical analysis was calculated by one-way ANOVA followed by the Student-Newman-Keuls test.

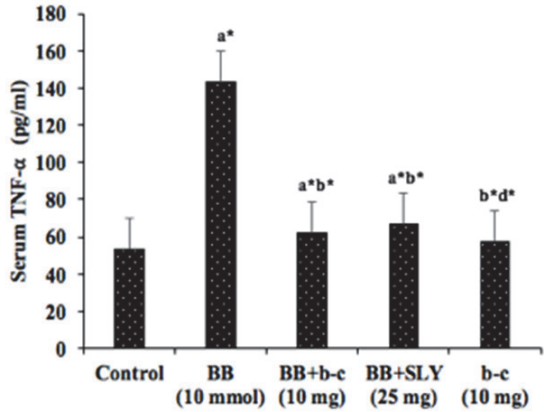

(A)

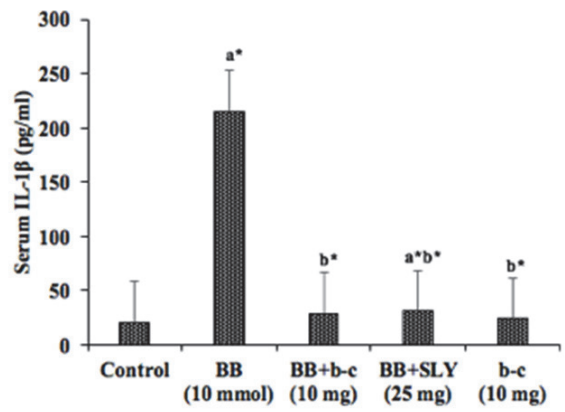

(B)

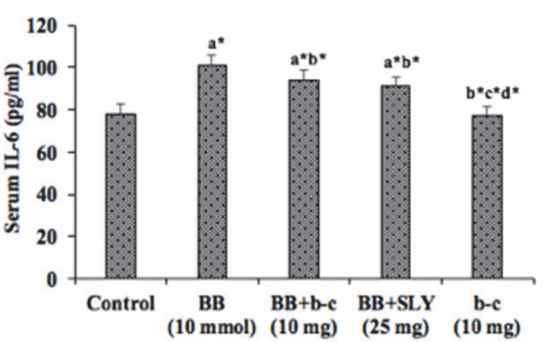

(C)

Fig. 8. Effect of beta-carotene on TNF- $\alpha$, IL- $1 \beta$ and IL-6 in serum of BB-induced rats. (A) SerumTNF- $\alpha$, (B) Serum IL-1 $\beta$, (C) Serum IL-6. Each value represents the mean \pm SE of six rats. Comparisons were made as follows: a - Group 1 vs Group 2, 3, 4, 5; b - Group 2 vs Group 3, 4, 5; c- Group 3 vs Group 4, 5; d - Group 4 vs Group 5. The symbols represent statistical significance at $\left({ }^{*} P<0.05\right)$. Statistical analysis was calculated by one-way ANOVA followed by the Student-Newman-Keuls test.

\section{Effect of beta-carotene on protein levels of Caspase-3, COX-2 and NF- $k B$ in liver of BB-induced rats}

The ratio of the target gene and housekeeping gene of group 1 were in normal range. The levels of Caspase- 3 , COX-2 and NF- $\kappa$ B were significantly elevated $\left({ }^{*} P<0.05\right)$ in BB-induced rats. The normalization of the enhanced levels of Caspase-3, COX-2 and NF- $\mathrm{KB}$ evoked by BB on pre-treatment with beta-carotene emphasizes the shielding effect of beta-carotene (Fig. 9). The group treated with silymarin showed protein levels comparable to those of the group treated with beta-carotene. Normal levels of Caspase-3, COX-2 and NF-kB were found in rats protected with beta-carotene alone.

\section{Discussion}

The liver is prone to toxicity from environmental pollutants, drugs and other xenobiotics as it has a major role in metabolism. BB causes local inflammation, mitochondrial dysfunction, ATP depletion and lipid peroxidation leading to loss of cellular integrity and function (Miller et al., 1990). Oxidative stress and free radicals are reported to induce activation and proliferation of hepatic stellate cells in the injured liver leading to the progression of chronic liver diseases (Ezhilarasan, 2018). Beta-carotene is known to be an antioxidant and has 


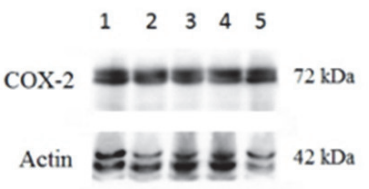

(a)

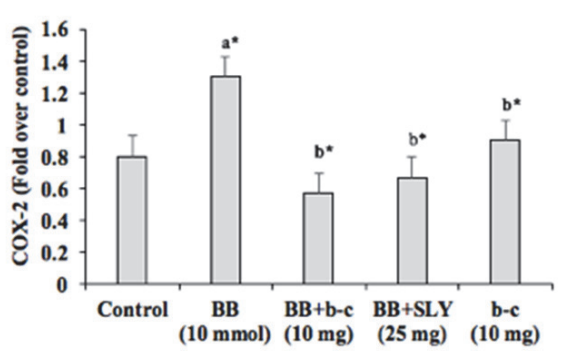

(A)

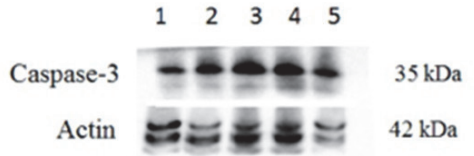

(b)

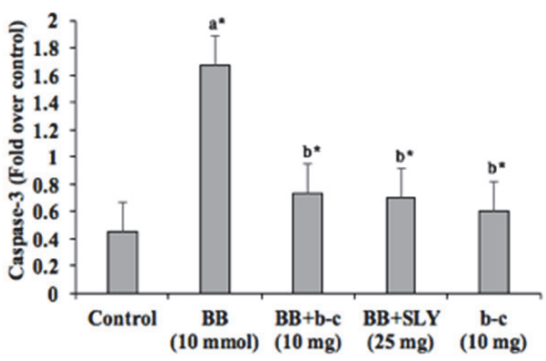

(B)

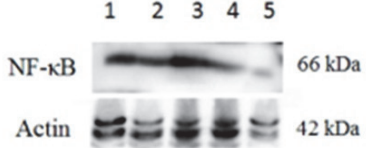

(c)

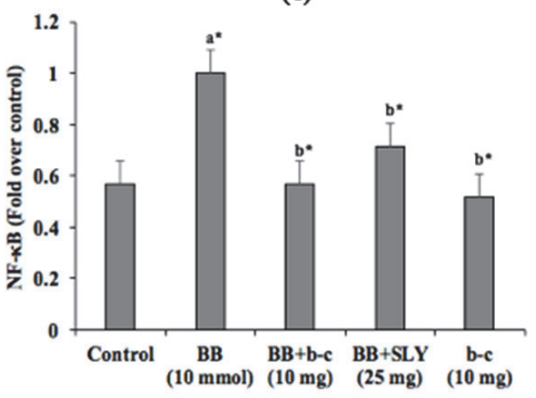

(C)

Fig. 9. Effect of beta-carotene on protein levels of Caspase-3, COX-2 and NF- $\kappa B$ in liver of BB-induced rats. Western blot analysis with actin as control: (A) COX-2, (B) Caspase-3, (C) NF-kB. Quantitative analysis of protein levels normalized to the control, which is represented in fold difference with the mean \pm SE of six rats. Comparisons were made as follows: a - Group 1 vs Group 2, 3, 4, 5; b - Group 2 vs Group 3, 4, 5; c - Group 3 vs Group 4, 5; d - Group 4 vs Group 5. The symbols represent statistical significance at $\left({ }^{*} P<0.05\right)$. Statistical analysis was calculated by one-way ANOVA followed by the Student-Newman-Keuls test.

been demonstrated to exert biological and pharmacological activities. As formation of reactive oxygen species (ROS) is also one of the major mechanisms of BB induced liver injury, the antioxidant properties of beta-carotene have an important role in preventing liver injury by concomitant administration.

The marker enzymes which are primarily used to measure liver damage are ALP, AST and ALT. Necrosis or damage to membrane promotes the release of these enzymes into circulation and results in increased levels of the same (Choudhary and Devi, 2014). Hepatocellular liver damage is characterized by an initial elevation in ALT and cholestatic liver damage by an initial elevation in ALP. As seen in our results, there was induction of severe liver damage in the rats treated with bromobenzene. This is evidenced by the increased levels of liver markers AST, ALT and ALP. The efficacy of beta-carotene in depreciating the levels of liver markers at comparable levels with the standard drug silymarin indicates that beta-carotene serves as an ameliorating agent for hepatocellular as well as cholestatic liver damage.

Activation of enzymes like CYP2E1 in the cytochrome P-450 system causes oxidative stress which plays a key role in liver damage. The injury inflicted on liver is enhanced further due to injury to bile duct cells and hepatocytes which in turn stimulates the accumulation of bile acid in the liver (Abou Seif, 2016). In our results, there was an increase in bilirubin in $\mathrm{BB}$-induced rats, which may be due to the enhanced production of bilirubin in concert with other factors as observed in aspartame induced toxicity (Choudhary and Devi, 2014). The direct bilirubin was brought down to normal levels in beta-carotene pre-treated rats showing its protective effect against oxidative stress. Studies have shown that exposure to xenobiotics induced variations in the levels of inflammatory cytokines like TNF- $\alpha$ and IL-1 $\beta$. These were associated with elevation in serum triglycerides, total cholesterol, VLDL cholesterol, and LDL cholesterol by inducing hepatic lipogenesis and inhibiting lipolysis and fatty acid oxidation (Yang et al., 2013). This is in correlation with our findings which showed a significant increase in triglycerides in the $\mathrm{BB}$ induced rats which were brought down in rats pre-treated with beta-carotene. Hypo- proteinaemia induced by BB was brought back to normalcy on pre-treatment with beta-carotene in the present study. The decrease in levels of albumin in rats induced with $\mathrm{BB}$ could be due to damage in liver caused by BB as anabolism of albumin takes place in liver. Beta-carotene could have a role in inhibiting damage to liver by its protective action thereby facilitating anabolism of albumin and proteins. The depletion of antioxidants like catalase, SOD, GST, GPx and GSH showed that there is oxidative stress and that liver damage is due to depleted antioxidant potential in the rats induced by BB. When there is an increase in ROS levels and concomitant fall in the antioxidant system, it leads to a variation in mitochondrial permeability and transition potential which sequentially induces the release of pro-apoptotic factors like cytochrome c (Wang et al., 2013). The significant increase in the antioxidant levels in BB-induced rats pre-treated with beta-carotene emphasizes the fact that the antioxidant and antiapoptotic property of beta-carotene plays a role in protecting against hepatotoxicity induced by BB.

The GSH pool is depleted due to the conjugation of epoxides formed by biotransformation of BB, when it is delivered in high doses (Kluwe et al., 1984). Hepatotoxicity induced by $\mathrm{BB}$ is characterized by a depletion in glutathione in both mitochondria and cytosol, succeeded by a diminution in mitochondrial respiration at complexes I and II during the start of hepatotoxicity (Vedi and Sabina, 2016). Pre-treatment with beta-carotene significantly increased the reduced glutathione levels brought about by the action of BB in our study. This result confirms that beta-carotene has the capacity to revive the GSH pool drained away by conjugation of epoxides of bromobenzene during biotransformation, thus enhancing the protective shield against ROS. The increased lipid peroxidation was evidenced in our results showing a steep rise in TBARS values in BB-induced rats. Pre-treatment with beta-carotene significantly reduced lipid peroxidation in par with silymarin treated rats and brought it down to normal values. The increase in GSH levels and the decrease in TBARS values leads us to conclude that beta-carotene plays a protective role against ROS thus increasing free radical scavenging and reducing oxidative stress, which culminates in the reduction of lipid perox- 
idation thereby controlling the biochemical events leading to hepatotoxicity.

Oxidative stress induces cytokines which further modulate formation of chemokines and induction of adhesion molecules in liver (Jaeschke et al., 2002). This culminates in cell necrosis due to release of proteases combined with degranulation of primed neutrophils which migrate and adhere to hepatocytes on receiving a chemotactic signal by chemokines and lipid peroxidation products, which are produced during the process of cell injury from parenchyma (Jaeschke, 2000). This explains the underlying cascade of events to our findings that $\mathrm{BB}$ treatment induces inflammation and cellular damage in hepatic tissue. A great number of the chronic diseases of the liver which ends in cirrhosis and fibrosis is preceded by inflammation. Pre-treatment with beta-carotene helped in normalizing hepatocyte architecture by regularizing biliary hyperplasia and cellular necrosis through regeneration.

Reactive secondary metabolites produced during biotransformation stimulate pro-fibrogenic cytokines, inflammatory markers and collagen synthesis during the development of hepatic fibrosis (Ezhilarasan, 2018). It has been reported that inflammatory status, in particular IL-6 levels, appears to be negatively associated with plasma beta-carotene levels (Rodríguez-Rodríguez et al., 2017). IL-1 $\alpha$, IL-1 $\beta$, TNF- $\alpha$ are the cytokines which are pro-inflammatory and production of these and activation of immune cells which are inherent are typical features of inflammation. This was supported by the elevation of cytokines TNF- $\alpha$, IL- $1 \beta$ and IL- 6 in BB induced rats in our experimental studies. Pre-treatment with beta-carotene helped in depreciating the levels of cytokines TNF- $\alpha$, IL-1 $\beta$ and IL- 6 in liver and serum in rats induced with BB. This suggests the role of beta-carotene as an anti-inflammatory agent thereby preventing series of events leading to hepatotoxicity induced by $\mathrm{BB}$.

Beta-carotene has demonstrated to effectively reduce the progression of secondary injury events following spinal cord injury by inhibiting the activation of NF- $\kappa$ B pathway (Zhou et al., 2018).

Elevation in the levels of Caspase-3and TNF- $\alpha$ would denote oxidative stress (Kakita et al., 2009). NF- $\kappa B$ has demonstrated to be involved in the switch to an inflammatory state after oxidative stress under in-vitro conditions (Arena et al., 2018). Pro-inflammatory cytokines IL-1 $\beta$ and TNF- $\alpha$ activate COX-2 which induces inflammation. COX-2 has a contributory role in the activation in extra hepatic vascular dysfunction and inflammation (Nascimento et al., 2018). ROS activates mitochondrial apoptosis pathway through Caspase- 3 modulated by TNF- $\alpha$ (Aghvami et al., 2018). Oxidative stress, inflammatory and apoptotic markers (Niu et al., 2018) were substantially increased in injured cells on BB-induction and brought back to normal level following pre-treatment with beta-carotene. In our studies, activation of NF- $\mathrm{kB}$ was inhibited by beta-carotene. The protective mechanism of beta-carotene may involve inhibition of NF- $\mathrm{BB}$ signal transduction pathway and subsequent downregulation of inflammatory mediators as evidenced by reduction in expression of NF- $\kappa \mathrm{B}$ associated genes like COX-2 (Geeviman et al., 2018). In our result, BB-induced rats were observed to show significant elevation in the level of Caspase-3, COX-2 and NF- $\kappa$ B. Beta-carotene was able to modulate and normalize the increased levels of Caspase-3, COX-2 and NF- $\kappa$ B in liver caused by BB. This demonstrates the ability of beta-carotene in protecting and preventing cellular damage caused by oxidative stress by modulating the apoptotic factors involved in cell signaling in concomitance with gene regulation leading to apoptosis.

\section{Conclusions}

The results of our present studies lead us to conclude that pre-treatment with beta-carotene in rats offers significant protection against bromobenzene-induced hepatotoxicity and oxidative stress comparable to silymarin, which is the standard drug of reference for hepatoprotection. Beta-carotene was shown to normalize the elevated liver function marker enzymes, lipid, total and direct bilirubin levels. Bromobenzene-induced reduction in antioxidant levels was restored to almost normal levels by concomitant administration of beta-carotene. Additionally, beta-carotene restored the normal architecture of hepatocytes through regeneration and decreased the generation of pro-inflammatory cytokines, including TNF- $\alpha$, IL- $1 \beta$ and IL- 6 emphasizing its anti-inflammatory properties. Furthermore, beta-carotene treatment markedly inhibited the NF- $\kappa \mathrm{B}$ pathway activation, COX-2 and Caspase-3 leading us to conclude that beta-carotene might have its protective role by inhibiting these vital steps in the signaling cascade pathways involved in regulation of cell death induced by metabolic imbalance. However, further pharmacological evidence supporting the role of beta-carotene against bromobenzene-induced liver injury is needed and more studies are required to explore the mechanisms for the same.

\section{Conflict of interests}

The authors declare that they have no conflict of interests.

\section{Acknowledgements}

The authors are thankful to Vellore Institute of Technology, Vellore, India for providing the necessary facilities to carry out this research project.

\section{References}

Abou Seif HS (2016). Physiological changes due to hepatotoxicity and the protective role of some medicinal plants. Beni-Suef Univ J Basic Appl Sci 5: 134-146. DOI: 10.1016/j.bjbas.2016.03.004.

Aghvami M, Salimi A, Eshghi P, Zarei MH, Farzaneh S, Sattari F, et al. (2018). Targeting the mitochondrial apoptosis pathway by a newly synthesized COX-2 inhibitor in pediatric ALL lymphocytes. Future Med Chem 10(19): 2277-2289. DOI: 10.4155/fmc-2018-0032.

Arena A, Zimmer TS, van Scheppingen J, Korotkov A, Anink JJ, Mühlebner A, et al. (2018). Oxidative stress and inflammation in a spectrum of epileptogenic cortical malformations: molecular insights into their interdependence. Brain Pathol Zurich Switz 29(3): 351-365. DOI: 10.1111/bpa.12661.

Avraham Y, Berry EM, Donskoy M, Ahmad WA, Vorobiev L, Albeck A, Mankuta D (2017). Beta-carotene as a novel therapy for the treatment of "Autistic like behavior" in animal models of Autism. Behav Brain Res 364: 469-479. DOI: 10.1016/j.bbr.2017.09.041.

Bahadır A, Ceyhan A, Öz Gergin Ö, Yalçın B, Ülger M, Özyazgan TM, Yay A (2018). Protective effects of curcumin and betacarotene on cisplatin-induced cardiotoxicity: An experimental rat model. Anatol J Cardiol 19: 213-221. DOI: 10.14744/ AnatolJCardiol.2018.53059.

Chen J, Jiang W, Shao L, Zhong D, Wu Y, Cai J (2016). Association between intake of antioxidants and pancreatic cancer risk: a meta-analysis. Int J Food Sci Nutr 67: 744-753. DOI: 10.1080/09637486.2016.1197892.

Choudhary AK, Devi RS (2014). Serum biochemical responses under oxidative stress of aspartame in wistar albino rats. Asian Pac J Trop Dis 4: S403-S410. DOI: 10.1016/S2222-1808(14)60478-3.

Darwish WS, Ikenaka Y, Nakayama S, Mizukawa H, Thompson LA, Ishizuka M (2018). $\beta$-carotene and retinol reduce benzo[a]pyreneinduced mutagenicity and oxidative stress via transcriptional 
modulation of xenobiotic metabolizing enzymes in human HepG2 cell line. Environ Sci Pollut Res. Int 25: 6320-6328. DOI: 10.1007/ s11356-017-0977-z.

Ezhilarasan D (2018). Oxidative stress is bane in chronic liver diseases: Clinical and experimental perspective. Arab J Gastroenterol 19(2): 56-64. DOI: 10.1016/j.ajg.2018.03.002.

Feyissa T, Asres K, Engidawork E (2013). Renoprotective effects of the crude extract and solvent fractions of the leaves of Euclea divinorum Hierns against gentamicin-induced nephrotoxicity in rats. J Ethnopharmacol 145: 758-766. DOI: 10.1016/j. jep.2012.12.006.

Fried MW, Navarro VJ, Afdhal N, Belle SH, Wahed AS, Hawke RL, et al. (2012). Effect of silymarin (milk thistle) on liver disease in patients with chronic hepatitis $C$ unsuccessfully treated with interferon therapy: a randomized controlled trial. JAMA 308(3): 274-282. DOI: 10.1001/jama.2012.8265.

Fujisawa S, Murakami Y (2016). Eugenol and Its Role in Chronic Diseases. Adv Exp Med Biol 929: 45-66. DOI: 10.1007/978-3-31941342-6_3.

Geeviman K, Babu D, Prakash Babu P (2018). Pantoprazole Induces Mitochondrial Apoptosis and Attenuates NF- $\mathrm{KB}$ Signaling in Glioma Cells. Cell Mol Neurobiol 38(8): 1491-1504. DOI: 10.1007/s10571-018-0623-4.

Gopi S, Setty OH (2010). Beneficial effect of the administration of Hemidesmus indicus against bromobenzene induced oxidative stress in rat liver mitochondria. J Ethnopharmacol 127: 200-203. DOI: 10.1016/j.jep.2009.09.043.

Habig WH, Pabst MJ, Jakoby WB (1974). Glutathione S-transferases. The first enzymatic step in mercapturic acid formation. J Biol Chem 249(22): 7130-7139.

Hamed MA, El-Rigal NS, Ali SA (2013). Effects of black seed oil on resolution of hepato-renal toxicity induced bybromobenzene in rats. Eur Rev Med Pharmacol Sci 17: 569-581.

Heijne WHM, Slitt AL, van Bladeren PJ, Groten JP, Klaassen CD, Stierum RH, van Ommen B (2004). Bromobenzene-induced hepatotoxicity at the transcriptome level. Toxicol Sci 79: 411-422. DOI: $10.1093 /$ toxsci/kfh128.

Jaeschke H (2000). Reactive oxygen and mechanisms of inflammatory liver injury. J Gastroenterol Hepatol 15: 718-724. DOI: 10.1046/j.1440-1746.2000.02207.x.

Jaeschke H, Gores GJ, Cederbaum AI, Hinson JA, Pessayre D, Lemasters JJ (2002). Mechanisms of hepatotoxicity. Toxicol Sci 65: 166-176. DOI: 10.1093/toxsci/65.2.166.

Kakita H, Aoyama M, Hussein MH, Kato S, Suzuki S, Ito T, et al. (2009). Diclofenac enhances proinflammatory cytokine-induced nitric oxide production through NF- $\mathrm{kB}$ signaling in cultured astrocytes. Toxicol Appl Pharmacol 238: 56-63. DOI: 10.1016/j. taap.2009.04.014.

Kluwe WM, Maronpot RR, Greenwell A, Harrington F (1984). Interactions between Bromobenzene Dose, Glutathione Concentrations, and Organ Toxicities in Single- and MultipleTreatment Studies. Toxicol Sci 4: 1019-1028. DOI: 10.1093/ toxsci/4.6.1019.

Koriem KMM, Arbid MS (2018). Evaluating of $\beta$-carotene role in ameliorating of favism-induced disturbances in blood and testis. J Complement Integr Med 15(3). DOI: 10.1515/jcim-2017-0164.

Li S, Tan H-Y, Wang N, Zhang Z-J, Lao L, Wong C-W, Feng Y (2015). The Role of Oxidative Stress and Antioxidants in Liver Diseases. Int J Mol Sci 16: 26087-26124. DOI: 10.3390/ijms161125942.

Liu Z, Ren Z, Zhang J, Chuang C-C, Kandaswamy E, Zhou T, Zuo L (2018). Role of ROS and Nutritional Antioxidants in Human Diseases. Front Physiol 9: 477. DOI: 10.3389/fphys.2018.00477.

Ma E, Iso H, Yamagishi K, Ando M, Wakai K, Tamakoshi A (2018). Dietary Antioxidant Micronutrients and All-Cause Mortality: The Japan Collaborative Cohort Study for Evaluation of Cancer Risk. J Epidemiol 28(9): 388-396. DOI: 10.2188/jea.JE20170023.

Marklund S, Marklund G (1974). Involvement of the superoxide anion radical in the autoxidation of pyrogallol and a convenient assay for superoxide dismutase. Eur J Biochem 47(3): 469-474. DOI: 10.1111/j.1432-1033.1974.tb03714.x.

Miller NE, Thomas D, Billings RE (1990). Bromobenzene metabolism in vivo and in vitro. The mechanism of 4-bromocatechol formation. Drug Metab Dispos Biol Fate Chem 18(3): 304-308.

Moron M, Depierre J, Mannervik B (1979). Levels of glutathione, glutathione reductase and glutathione $S$-transferase activities in rat lung and liver. Biochim Biophys Acta 582: 67-78. DOI: 10.1016/0304-4165(79)90289-7.

Nascimento M, Piran R, Da Costa RM, Giordani MA, Carneiro FS, Aguiar DH, et al. (2018). Hepatic injury induced by thioacetamide causes aortic endothelial dysfunction by a cyclooxygenasedependent mechanism. Life Sci 212: 168-175. DOI: 10.1016/j. lfs.2018.09.051.

Nita M, Grzybowski A (2016). The Role of the Reactive Oxygen Species and Oxidative Stress in the Pathomechanism of the AgeRelated Ocular Diseases and Other Pathologies of the Anterior and Posterior Eye Segments in Adults. Oxid Med Cell Longev 2016: 3164734. DOI: 10.1155/2016/3164734.

Niu X, Zheng S, Liu H, Li S (2018). Protective effects of taurine against inflammation, apoptosis, and oxidative stress in brain injury. Mol Med Rep 18(5): 4516-4522. DOI: 10.3892/ mmr.2018.9465.

Ohkawa H, Ohishi N, Yagi K (1979). Assay for lipid peroxides in animal tissues by thiobarbituric acid reaction. Anal Biochem 95: 351-358. DOI: 10.1016/0003-2697(79)90738-3.

Rodríguez-Rodríguez E, López-Sobaler AM, Navia B, Andrés P, Jiménez-Ortega AI, Ortega RM (2017). $\beta$-Carotene Concentration and Its Association with Inflammatory Biomarkers in Spanish Schoolchildren. Ann Nutr Metab 71: 80-87. DOI: 10.1159/000479009.

Rotruck JT, Pope AL, Ganther HE, Swanson AB, Hafeman DG, Hoekstra WG (1973). Selenium: biochemical role as a component of glutathione peroxidase. Science 179: 588-590. DOI: 10.1126/ science.179.4073.588.

Sinha AK (1972). Colorimetric assay of catalase. Anal Biochem 47: 389-394. DOI: 10.1016/0003-2697(72)90132-7.

Vedi M, Sabina EP (2016). Assessment of hepatoprotective and nephroprotective potential of withaferin A on bromobenzeneinduced injury in Swiss albino mice: possible involvement of mitochondrial dysfunction and inflammation. Cell Biol Toxicol 32: 373-390. DOI: 10.1007/s10565-016-9340-2.

Vedi M, Rasool M, Sabina EP (2014a). Amelioration of bromobenzene hepatotoxicity by Withania somnifera pretreatment: Role of mitochondrial oxidative stress. Toxicol Rep 1: 629-638. DOI: 10.1016/j.toxrep.2014.08.009.

Vedi M, Rasool M, Sabina EP (2014b). Protective effect of administration of Withania somnifera against bromobenzene induced nephrotoxicity and mitochondrial oxidative stress in rats. Ren Fail 36: 1095-1103. DOI: 10.3109/0886022X.2014.918812.

Wang B, Van Veldhoven PP, Brees C, Rubio N, Nordgren M, Apanasets O, et al. (2013). Mitochondria are targets for peroxisome-derived oxidative stress in cultured mammalian cells. Free Radic Biol Med 65: 882-894. DOI: 10.1016/j. freeradbiomed.2013.08.173.

Wang XD, Russell RM (1999). Procarcinogenic and anticarcinogenic effects of beta-carotene. Nutr Rev 57: 263-272. DOI: 10.1111/ j.1753-4887.1999.tb01809.x.

Yang J, Liu D, Jing W, Dahms H-U, Wang L (2013). Effects of cadmium on lipid storage and metabolism in the freshwater crab Sinopotamon henanense. PloS One 8: e77569. DOI: 10.1371/ journal.pone.0077569.

Zhou L, Ouyang L, Lin S, Chen S, Liu Y, Zhou W, Wang X (2018). Protective role of $\beta$-carotene against oxidative stress and neuroinflammation in a rat model of spinal cord injury. Int Immunopharmacol 61: 92-99. DOI: 10.1016/j. intimp.2018.05.022. 